\section{Minociclina como alternativa en el tratamiento de pulpotomía de dientes temporales}

\section{Minocycline as an alternative in the treatment of pulpotomy in temporary teeth}

\section{Resumen}

Objetivo. Determinar la eficacia de la minociclina en gel de liberación prolongada siendo un agente antimicrobiano en el tratamiento de la pulpotomía infantil y la eficacia de la minociclina aplicada como medicamento en el tratamiento de pulpotomía. Métodos. A 39 órganos dentales se les realizó el tratamiento de pulpotomía de acuerdo a la técnica descrita por Sweet y cols., previo y posterior a la colocación del medicamento minociclina, se tomó una muestra (colocación de punta de papel) y se realizó conteo de microorganismos por método de conteo de placa. Resultados. Se realizó una revisión clínica y radiográfica, haciéndolo a los 15, 30, 90 y 180 días, los resultados se registraron de acuerdo al instrumento clínico y radiográfico de la Asociación Americana de Odontología Pediátrica (AAPD). Se presentó tres casos de fracaso que representa apenas el 8\%, teniendo un éxito de $92 \%$ este tratamiento. Conclusiones. Se concluyó que la medicación con minociclina resulta un auxiliar valioso en la desinfección local de la cámara pulpar y por tanto para el tratamiento de pulpotomías.

Palabras clave: Odontología; Pulpotomía; Minociclina (fuente: DeCS BIREME).

\section{Artículo Original}

José Eduardo Orellana Centeno ${ }^{1,2, a}$, Mauricio González Osorio ${ }^{2, b}$

${ }^{1}$ Universidad Autónoma de San Luis Potosí, Facultad de Enfermería y Nutrición, Maestría en Salud Pública, San Luis Potosí, México.

2 Universidad de la Sierra Sur, Instituto de Investigación en Salud Pública, Licenciatura de Odontología, Miahuatlán de Porfirio Díaz, Oaxaca, México.

a Maestro en Salud Pública.

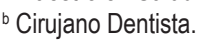

\section{Correspondencia:}

José Eduardo Orellana Centeno

Correo electrónico: jeorellana@unsis.edu.mx

Cárdenas No. 122. Fraccionamiento Los Sauces, Rioverde, San Luis Potosí, México.

\section{Coautores:}

Mauricio González Osorio

odonto.unsis@gmail.com

\section{Editora:}

María Antonieta Pérez Flores Universidad de Concepción, Chile.

Conflicto de intereses: los autores declaran no tener conflictos de interés.

Fuente de financiamiento: sin financiamiento.

Recibido: 20/11/18

Aceptado: 03/06/19

Publicado: $12 / 09 / 19$

\begin{abstract}
Objetive. To determine the effectiveness of the minocycline gel prolonged release as an antimicrobial agent in the treatment of pulpotomy in child and the effectiveness of the minocycline applied as a drug in the treatment of pulpotomy. Methods. Pulpotomy treatment was perfomed in 39 dental samples according to the technique described by Sweet et al., before and after the minocycline drug was placed. A sample was taken (paper tip placement) and microorganisms were counted, by plate counting method. Results. A clinical and radiographic review was performed at 15, 30, 90 and 180 days. The results were recorded according to the clinical and radiographic instrument of the American Pediatric Dentistry Association (AAPD). There were three cases of failure, representing only $8 \%$, with $92 \%$ success in this treatment. Conclusions. It was concluded that the medication with minocycline is a valuable auxiliary in the local disinfection of the pulp chamber and therefore for the treatment of pulpotomies.
\end{abstract}

Keywords: Dentistry; Pulpotomy; Minocycline (source: MeSH NLM). 


\section{Introducción}

En los últimos años, el uso de medicamentos, principalmente antibióticos, de liberación local se considera primordiales, dada las objeciones a los antibióticos sistémicos, ya que si en un tratamiento necesitamos mayor concentración debemos aumentar la dosis, por lo que el riesgo de efectos adversos se incrementa, aspecto que se minimiza con la aplicación local de antibióticos ${ }^{1}$.

Los antibióticos son sustancias de origen natural, sintética o semisintetica que actúan inhibiendo los microorganismos a una dilución elevada y ejercen su acción a nivel molecular en un proceso metabólico o en una estructura concreta de una microorganismo ${ }^{2}$.

Los agentes antibacterianos se pueden dividir en dos grupos, de acuerdo a su acción: bacteriostáticos y bactericidas, estos últimos se logran cuando existe la eliminación de todos los microorganismos sensibles ${ }^{3}$.

En los procedimientos endodónticos, existen bases elementales para utilización de los antibióticos. En efecto algunos de estos criterios son los indicadores de la antibioticoterapia, lo que indica que el uso de antibióticos puede no corresponder a una terapéutica definitiva. Es necesario la realización del tratamiento endodóntico adecuado, para prevenir la colonización y la difusión de las bacterias y sus productos, desde el conducto a los tejidos periapicales. El propósito principal de la administración de un antibiótico en estos casos es: limitar la difusión de la infección, tratar la infección sistémica y ayudar a la resolución de los síntomas. Otro de los aspectos que se maneja es la elección del antibiótico, esto depende del microorganismo, del huésped y del propio antibiótico ${ }^{4}$.

También se debe pensar en los efectos secundarios, aunque los antibióticos actúen de forma selectiva contra los microorganismos, no está exento el paciente de sufrir efectos indeseables, que pueden ser de tres tipos: tóxicos, por la lesión de determinadas estructuras celulares del huésped; alérgicos, que dan lugar a reacciones de hipersensibilidad tipo I; y biológicos, como las destrucción de la microbiota normal ${ }^{5}$.

Los antibióticos deben suspenderse después de terminar la terapia y nunca debe hacerse en dosis decrecientes ya que esto aumenta el riesgo de generar resistencias por parte de los microorganismos ${ }^{6}$.

El uso de la minociclina en la Odontología es relativamente reciente ya que se ha utilizado en el tratamiento de las bolsas periodontales previo al raspado y alisado radicular para mejorar el pronóstico del tratamiento periodontal, cuyo objetivo primario es detener la progresión de la enfermedad ${ }^{7}$.

En estudios de terapias de liberación local se ha incluido el uso de tetraciclina, doxiciclina, metronidazol y minociclina, siendo esta última señalada como uno de los antibióticos más efectivos para microorganismos asociados con periodontitis. Se ha descrito una disminución considerable de Porphyromonas gingivalis y Bacteroides forsythus, teniendo resultados favorables a una semana de ser aplicado, como tratamiento único sin efectuar raspado y alisado radicular ${ }^{8}$.

Las tetraciclinas han demostrado ser el antibiótico con la más alta liposolubilidad, por lo que es el agente antimicrobiano más adecuado para el control de la enfermedad periodontal, especialmente cuando es empleado como terapia local ${ }^{9}$.

La minociclina es un bactericida contra microorganismos Gram positivos y negativos; una amplia gama de microorganismos periodontales son susceptibles a la minociclina, entre ellos microorganismos gram positivos (Actinomyces, Peptostreptococcus, Propionibacterium, Eubacteria y Lactobacillus spp) y en esencia todos los microorganismos relacionados con la enfermedad periodontal (A. actinomycetemcomitans, P. gingivalis, Eikenella corrodens y Campylobacter rectus). Una excepción es $\mathrm{P}$. intermedia que tiene algunas cepas resistentes a las tetraciclinas ${ }^{10}$.

Las tetraciclinas presentan su actividad antimicrobiana dentro del saco periodontal y la dentina. Se presenta con altas concentraciones al aplicarse de forma local, mejoran sus propiedades y se inhibe el desarrollo de especies resistentes. La liberación controlada, permite que la concentración sea mantenida por varios días reduciendo el desarrollo de especies resistentes ${ }^{11}$.

Para la minociclina se han desarrollado tres formas de aplicación local: en película, microencapsulada y en ungüento ${ }^{12}$.

La minociclina se acumula en superficies radiculares y hueso permitiendo a estos sitios actuar como reservorios de una baja pero activa liberación de concentraciones efectivas de la droga por varios días, lo que permite ejercer su acción antimicrobiana y anticolagenasa en sitios infectados por un largo periodo de tiempo. Las desventajas que presenta son el riesgo de causar pigmentación en piel y pigmentación intraoral. El mecanismo de acción, es la de un bactericida de amplio espectro que inhibe la síntesis proteínica bacteriana ${ }^{13}$.

El propósito de este estudio es determinar la eficacia de la minociclina en gel de liberación prologada aplicada como medicamento en el tratamiento de pulpotomías de dientes infantiles.

\section{Métodos}

El tipo de estudio fue cuantitativo, cuasiexperimental, analítico y prospectivo. El universo poblacional fue obtenido de pacientes que acudieron a la Clínica Odontopediatría de la Facultad de Estomatología de la Universidad Autónoma de San Luis Potosí, se utilizaron 39 órganos dentales en los que previamente se había establecido diagnóstico de pulpotomía de 39 pacientes (20 del sexo femenino y 19 del masculino, con un rango de edad entre los 4 y 8 años), que se puede observar en la tabla 1. El diagnóstico fue confirmado clínica y radiográficamente en los órganos dentales revisados, y que se cumplía con los criterios de inclusión para el tratamiento de pulpotomía y del estudio. Se solicitó autorización 
a los padres de familia explicándoles el procedimiento a realizar y se obtuvo su firma en la carta de consentimiento informado.

Tabla 1. Características de la muestra según edad, sexo e índice ceod

\begin{tabular}{llll}
\hline \multicolumn{1}{c}{ Variable } & \multicolumn{1}{c}{ Media +/- DE } & $\begin{array}{c}\text { Mínimo- } \\
\text { máximo }\end{array}$ & n (\%) \\
\hline Edad & $8.67+/-2.36$ & $(4-8)$ & \\
Sexo & & & $20(52)$ \\
Femenino & & & $19(48)$ \\
Masculino & & & \\
Índice ceod & $2.15+/-1.43$ & $(0-20)$ & \\
Cariados & $4.45+/-3.01$ & $(0-9)$ & \\
Obturados & $2.46+/-1.69$ & $(0-6)$ & \\
Extraídos & $4.39+/-2.69$ & $(0-9)$ & \\
\hline
\end{tabular}

Criterios de inclusión. Se consideraron los siguientes criterios de inclusión:

- Pacientes con edad entre 4 y 9 años

- Dientes superiores o inferiores, anteriores o posteriores con diagnóstico presuntivo de pulpotomía

- Dientes con caries profunda sin exposición pulpar

- Conservación de longitud radicular en más de $2 / 3$

- Dientes con posibilidad de restauración luego del tratamiento de pulpotomía.

Criterios de exclusión. Se consideraron los siguientes criterios de exclusión:

- Pacientes sistémicamente comprometidos o con algún tipo de síndrome

- Terapia antibiótica previa

- Infección dental

- Lesión en furca

- Reabsorción interna

- Reabsorción externa

- Degeneración pulpar

- No presenta sangrado al comunicar la cámara pulpar

- Pacientes con hemorragia no controlable.

- Pacientes con hemorragia con exudado purulento.

Criterios de eliminación. Se consideraron como criterios de eliminación:

- Pacientes que al momento del tratamiento endodóntico presenten el diente necrótico

- Realización de accidentes como perforación de furca

- Pacientes que no acudan a sus citas de seguimiento

Procedimientos. A los 39 órganos dentales que cumplieron con los criterios anteriormente señalados, se les realizó el tratamiento de pulpotomía de acuerdo a la técnica descrita por Sweet y cols. ${ }^{14}$ que normalmente se desarrolla para dicha terapia pulpar, con la excepción de que previo a la colocación del medicamento se tomó una muestra (colocación de punta de papel que se utiliza para secado en conductos previamente esterilizada dentro de la cámara pulpar) y posteriormente la colocación del medicamento, así como también se modificó el medicamento que normalmente se utiliza, que es formocresol, y en este caso se sustituyó con minociclina, la cantidad que fuera suficiente para cubrir el piso de la cámara pulpar. Se volvió a tomar una muestra de igual forma utilizando punta de papel y se llevó a laboratorio para analizar la muestra, se utilizaron técnicas de sepsia y antisepsia para una adecuada toma de muestra y para su transporte se empleó el método de Stuart, se utilizó tubo de ensayo previamente estéril, con tioglicolato. En el laboratorio se realizó conteo de microorganismos por método de conteo de placa.

La minociclina usada en este estudio se encuentra disponible comercialmente como un gel de clorhidrato de minociclina al 2,0\% llamado Periofeel Dental Oint. Contiene: $20 \mathrm{mg}$ de celulosa de hidroxietil, $25 \mathrm{mg}$ de cloruro de magnesio, $10 \mathrm{mg}$ de Eudragit RS, $6 \mathrm{mg}$ traisetina y glicerina de $0,5 \mathrm{~g}$ contenidas en un aplicador de polipropileno. Cada aplicación de $0,5 \mathrm{~g}$ de ungüento contiene el equivalente a $10 \mathrm{mg}$ de minociclina.

Se realizó una revisión clínica y radiográfica a los 15, 30, 90 y 180 días, los resultados se registraron de acuerdo al instrumento clínico y radiográfico de la Asociación Americana de Odontología Pediátrica (AAPD).

Análisis de datos. La información se analizó mediante estadística descriptiva y se apoyó mediante el paquete estadístico IBM SPSS versión 20 para Windows. Se aplicaron frecuencias, porcentajes, medidas de tendencia central y de dispersión.

\section{Resultados}

Se presentó tres casos de fracaso que representa apenas el $8 \%$, teniendo un éxito de tratamiento del $92 \%$. Dentro de los resultados que se observaron clínicamente fueron dolor constante posterior a la pulpotomía y radiológicamente se observó reabsorción externa y una disminución en la longitud radicular en el órgano dental tratado (Tablas 2 y 3). Se realizó el conteo de microorganismos en el laboratorio con las muestras y se presentan los resultados obtenidos en la tabla 4 .

\section{Discusión}

El objetivo biológico de los procedimientos de limpieza consiste en eliminar todo el tejido pulpar, las bacterias y sus toxinas de la cámara pulpar, esto con el fin de poder eliminar la pulpa cameral y permitir que la pulpa remanente de conductos permanezca y mantenga sus funciones dentro del diente.

En la actualidad se presenta la polémica sobre el uso del formocresol; este material ha sido muy cuestionado por presentar reacciones adversas, pero a pesar de esto, sigue 
siendo el material más empleado en los procedimientos de pulpotomías.

El formocresol presenta efecto cancerígeno (reportado en estudios con primates y perros de laboratorio), así como se sabe de sus efectos embriotóxicos y teratógenos. Como consecuencia de esto, en odontología, la decisión para dejar de utilizar el formocresol de parte de muchos especialistas se basa en la publicación que hizo la International Agency for Research on Cancer (IARC) en junio del 2004. En este reporte se dice que el vapor del formaldehido es un carcinógeno para los humanos. La extensiva y sistemática revisión concluyó que el formaldehido tiene relación positiva con el carcinoma nasofaríngeo y posiblemente con otros sitios del tracto respiratorio alto como la mucosa nasal y senos paranasales.

Tabla 2. Resultados de fracasos de la evaluación clínica a 180 días de las dos terapias pulpares

\begin{tabular}{cc}
\hline Criterios clínicos & Pulpotomía \\
\hline Absceso & $0(0 \%)$ \\
Trayecto fistuloso & $0(0 \%)$ \\
Movilidad & $0(0 \%)$ \\
Dolor a la percusión & $0(0 \%)$ \\
Dolor constante & $3(8 \%)$ \\
Cambio de coloración & $0(0 \%)$ \\
Inflamación de la encía & $1(3 \%)$ \\
\hline
\end{tabular}

Los tratamientos endodónticos parciales se indican en la mayoría de los casos fundamentalmente en dientes vitales; sin embargo, el principal objetivo de las pulpotomías con formocresol en dientes temporales ha sido su mantenimiento asintomático hasta su normal exfoliación, evitando así las pulpectomías totales que siempre son más complicadas debido a la morfología característica de los dientes temporales y la cooperación siempre comprometida de los pacientes infantiles.

Distintos autores como Tobón, Morawa, Furs, García Godoy, Loos y otros, plantean que diluyendo el formocresol puede reducirse su toxicidad, cuando se aplica durante $5 \mathrm{~min}$ en pulpas vitales de dientes temporales, lo que previene el daño a las capas profundas.

Uno de los tratamientos más populares en molares primarios cuando se produce exposición pulpar por caries, en ausencia de signos y síntomas de patología pulpar, es la pulpotomía con formocresol; a partir del manejo y conocimiento de los inconvenientes de utilizarlo, se comenzaron a buscar algunas otras sustancias o técnicas.

El glutaraldehído es una alternativa química, que ha sido propuesta para el tratamiento de las pulpotomías en dientes primarios, y ha recibido particular atención como sustituto del formocresol, porque es un fijador suave y potencialmente menos tóxico.

El hidróxido de calcio, fue una de las primeras sustancias que se utilizaron para el tratamiento de pulpotomía, es uno de los medicamentos con mayores usos dentro de la odontología por su biocompatibilidad y cumplen los requerimientos de una suspensión que debe ser usada como un recubridor, desarrollando un puente de dentina, con solo el único inconveniente de desarrollar reabsorción.

Dentro de las sustancias o medicamentos utilizados de manera alternativa, se encuentran los hemostáticos, uno de ellos es el sulfato férrico que se ha utilizado al 15,5\% (Astringedent) y actualmente al 20\% (Viscostat). Este compuesto de hierro se utiliza por su acción fuertemente hemostática y su efecto bactericida moderado, pero no tiene acción fijadora de tejidos o momificante. Otra de las sustancias con esa característica es el cloruro de aluminio, que reduce rápidamente la hemorragia pulpar; al entrar en contacto con la sangre forma un complejo proteínico y la membrana en este complejo sella los vasos, adicionando que tiene características bactericidas nos permiten una cavidad completamente limpia y estéril, además de que nos permite tener una pulpa radicular remanente completamente intacta y permitiendo que la pulpa haga sus funciones normales de defensa y de formación.

Una de las sustancias que recientemente se han utilizado como alternativa en la terapia de la pulpotomía es el MTA (trioxido mineral agregado), el cual es un cemento muy prometedor en el campo de la endodoncia y de la odontopediatría. Autorizado por la Food and Drug Administration comenzó a utilizarse en pulpotomías de dientes temporales. El preparado que se emplea es el gris, dado que el blanco tiene pobres resultados.

Es por ello la necesidad de buscar alternativas en el tratamiento de pulpotomías en dientes temporales y por esa razón la opción de un antibiótico como lo es la minociclina. Otro tipo de antibióticos, como el caso de ungüento oftálmico, que también por tener varios antibióticos de amplio espectro han tenido resultados muy alentadores.

Tabla 3. Resultados de fracasos de la evaluación radiográfica a 180 días de las dos terapias pulpares

\begin{tabular}{cc}
\hline Criterios radiográficos & Pulpotomía \\
\hline Lesión periapical & $0(0 \%)$ \\
Lesión de furca & $0(0 \%)$ \\
Ensanchamiento de ligamento & $0(0 \%)$ \\
Reabsorción interna & $1(3 \%)$ \\
Reabsorción externa & $2(5 \%)$ \\
Degeneración pulpar & $0(0 \%)$ \\
Longitud radicular & $2(5 \%)$ \\
Rarefacción ósea & $0(0 \%)$ \\
Osteorarefacción interradicular & $0(0 \%)$ \\
Calcificación pulpar & $0(0 \%)$ \\
\hline
\end{tabular}

En cuanto a tasa de éxito radiográfico reportado en pulpotomías podemos encontrar que en el estudio de Flaitz y cols. (72\% vs $85,07 \%)$. En ese mismo sentido Coll y cols., en un estudio retrospectivo reportaron tasas de 
éxito de pulpotomías en dientes temporales anteriores ( $86 \%$ vs $85,07 \%)$. Comparando los resultados obtenidos con los presentados por Flatiz y cols., son superiores al $85 \%$ que ellos reportan, aunque no utilizaron el mismo instrumento de medición y se basaron en el fundamento clínico.

Tabla 4. Resultados de conteo de microorganismos en las muestras.

\begin{tabular}{|c|c|c|}
\hline Paciente & $\begin{array}{l}\text { Medición Pre } \\
\text { (UFC/ml) }\end{array}$ & $\begin{array}{l}\text { Medición Post } \\
\text { (UFC/ml) }\end{array}$ \\
\hline 1 & 3 & 0 \\
\hline 2 & 2 & 1 \\
\hline 3 & 4 & 0 \\
\hline 4 & 5 & 0 \\
\hline 5 & 6 & 0 \\
\hline 6 & 9 & 0 \\
\hline 7 & 8 & 1 \\
\hline 8 & 5 & 0 \\
\hline 9 & 4 & 0 \\
\hline 10 & 6 & 0 \\
\hline 11 & 7 & 2 \\
\hline 12 & 4 & 0 \\
\hline 13 & 6 & 0 \\
\hline 14 & 7 & 1 \\
\hline 15 & 5 & 0 \\
\hline 16 & 8 & 1 \\
\hline 17 & 6 & 1 \\
\hline 18 & 3 & 0 \\
\hline 19 & 4 & 0 \\
\hline 20 & 5 & 0 \\
\hline 21 & 8 & 1 \\
\hline 22 & 9 & 3 \\
\hline 23 & 2 & 1 \\
\hline 24 & 3 & 0 \\
\hline 25 & 4 & 2 \\
\hline 26 & 3 & 2 \\
\hline 27 & 4 & 1 \\
\hline 28 & 3 & 1 \\
\hline 29 & 4 & 0 \\
\hline 30 & 5 & 1 \\
\hline 31 & 3 & 0 \\
\hline 32 & 5 & 1 \\
\hline 33 & 2 & 0 \\
\hline 34 & 3 & 1 \\
\hline 35 & 6 & 3 \\
\hline 36 & 2 & 0 \\
\hline 37 & 3 & 1 \\
\hline 38 & 3 & 0 \\
\hline 39 & 2 & 0 \\
\hline Media \pm DE & $4,6 \pm 2,0$ & $0,6 \pm 0,8$ \\
\hline
\end{tabular}

Aminabadi y cols., en un estudio clínico aleatorizado, reportaron radiográficamente tasa de éxito de $76 \%$ para pulpotomías con formocresol y $91 \%$ para pulpectomía, utilizo una baja concentración para formocresol (solución al $1,5 \%$ ) que es diferente a la que normalmente se utiliza en la técnica estándar de Sweet y además la restauración del diente tratado fue con resina autocurable. Estos resultados difieren de la presente investigación, en la que se obtuvo una tasa de éxito superior al 90\%, utilizando formocresol como medicamento mediante la técnica de Sweet; no se utilizó el mismo instrumento para medir y el tipo de restauración que utilizaron no es el más recomendable, debió haber sido corona; una de las ventajas de este estudio es que fue un ensayo clínico aleatorizado.

Casas y cols., en un esfuerzo de utilizar una técnica de terapia pulpar diferente al uso de formocresol, realizó la pulpotomía con sulfato férrico reportando una tasa de éxito del $79 \%$ para pulpotomía y $82 \%$ para pulpectomía. La utilización de una sustancia como es el sulfato férrico (hemostático) y minociclina, les da la ventaja de haber sido utilizado en otras áreas de la Odontología, aunque reportan resultados más bajos el sulfato férrico comparados con la minociclina.

El porcentaje de efectividad de la minociclina es significativamente mayor para eliminar bacterias presentes en las cámaras pulpares y por ende para el tratamiento de pulpotomía. Estos resultados son importantes en el ámbito endodóntico ya que son pocos los estudios en los cuales se haya administrado la minociclina como medicamento intraconducto. Las bacterias aisladas encontradas en los conductos necróticos son sensibles a la minociclina en gel de liberación prolongada.

Se recomienda realizar más estudios en esta misma línea de investigación pero utilizando un tamaño de muestra mayor, incluir un grupo control, aleatorizar los participantes del estudio, realizar no solamente un conteo de UFC/ml, sino que también identificar el tipo de microorganismos presentes.

Los resultados de este estudio in vivo demuestran que la minociclina en gel de liberación prolongada, a las 24 horas de aplicada, tiene una gran actividad bactericida, es capaz de reducir la presencia bacteriana, dando un mejor pronóstico del tratamiento. Se concluyó que la medicación para el tratamiento de pulpotomía en dientes infantiles con minociclina resulta ser un auxiliar valioso en la desinfección local del sistema de conductos radiculares.

\section{Referencias bibliográficas}

1. Waterhouse PJ. "New age" pulp therapy: Personal thoughts on a hot debate. Pediatr Dent. 2008;30:247-52.

2. Yeom HR. Clinical and microbiological effects of minocycline loaded microcapsules in adult periodontitis. J. Periodontol. 2009;68:1102-1109.

3. Vandekerckhave B, Quirynen M. The use of locally delivered minocycline in the treatment of chronic periodontitis. A review of literature. J Clin. Periodontol. 2008;25:964-968.

4. Koshi S, Love RM. Endodontic treatment in the primary dentition. Aust Endod J. 2004;30:59-68.

5. Tikka TM, Koistinaho JE. Minocycline provides neuroprotection against N-methyl-D-aspartate neurotoxicity by inhibiting microglia. J. Inmunol. 2010;166(12):7527-33.

6. Rodriguez-Cordeiro M, Carvalho-Rocha MJ. The effects of periradicular inflamation and infection on a primary 
tooth and permanent sucesor. J. Clin Pediatr Dent. 2008;29(3):193-200.

7. LaPorta VNJ. Minocycline-associated intra-oral soft-tissue pigmentation: clinicopathologic correlations and review. Journal of Clinical Periodontology 2005;32:119-122.

8. Greentein, G. Clinical Significance of Bacterial Resistance to Tetracyclines in the Treatment of Periodontal Diseases. Journal of Clinical Periodontology 1995;925-932

9. Seymour R, Heasman P. Tetracyclines in the management of periodontal diseases. J.Clin. Periodontol. 2009;22:22-35.

10. McColl E. Supportive periodontal therapy using mechanical instrumentation or $2 \%$ minocycline gel: a 12 month randomized, controlled, single masked pilot study. Journal of Clinical Periodontology 2006;33:141-150.

11. Graca MA. A randomized controlled trial of a $2 \%$ minocycline gel as an adjunct to non-surgical periodontal treatment, using a design with multiple matching criteria. Journal of Clinical Periodontology 1997;24:249-253.

12. Radva RM. Comparison of 3 Periodontal Local Antibiotic Therapies in Persistent Periodontal Pockets. Journal of Clinical Periodontology 1996;860-865.

13. Schenkein H. The Pathogenesis of Periodontal Diseases. Journal of Clinical Periodontology 1999;70:457-470.

14. Sweet CA, et al. Procedure for treatment of exposed and pulpless deciduous teeth. Journal of the American Dental Association 1930; 17(6): 1150-1153.

15. Boeve Z, Dermaut G. Clinical and radiographic comparison of primary molars after formocresol and electrosurgical pulpotomy: a randomized clinical trial. Indian J Dent Res 2008;19:219-23.

16. AAPD. Guideline on pulp therapy for primary and young permanent teeth. Pediatr. Dent. 2008;30:170-4.

17. Tobón G. Endodoncia Simplificada. 2da. ed. Cali: OPS; 1981.

18. Furs AB. Clinical evaluation of diluted formocresol pulpotomies in primary school children. Pediatr Dent 2010;3:321.

19. García-Godoy F. Pulp tissue reaction to diluted formocresol histologic study in dogs. Rev Dent (Sto Domingo) 2009;20:15.
20. Fuks AB. Current concepts in vital primary pulp therapy. Eur. J. Paediatr. Dent. 2002;3:115-20.

21. Avram DC, Pulver F. Pulpotomy medicaments for vital primary teeth. Surveys to determine and attitudes in pediatric dental practice and in dental school throught the world. J Dent Child 1989;56(6):426-34.

22. Calatayud J, Casado I, Álvarez C. Análisis de los estudios clínicos sobre la eficacia de las técnicas alternativas al formocresol en las pulpotomías de dientes temporales. Av Odontoestomatol. 2006;22(4):229-239.

23. Casas MJ, Kenny DJ, Judd PL, Johnston DH. Do we still need formocresol in pediatric dentistry? J. Can. Dent. Assoc. 2005;71:749-51.

24. Orellana JE, González JS, Nava JF, Olvera N, Orellana $M$, Ponce S. "Cloruro de aluminio una alternativa en pulpotomías de dientes temporales". Revista Latinoamericana de Ortodoncia y Odontopediatria "Ortodoncia.ws" edición electrónica marzo 2012. Obtenible en: www.ortodoncia.ws. Consultada 21 de Abril de 2019.

25. Zealand CM, Briskie DM, Botero TM, Boynton JR, $\mathrm{Hu}$ JC. Comparing gray mineral trioxide aggregate and diluted formocresol in pulpotomized human primary molars. Pediatr. Dent. 2010;32: 393-9.

26. Orellana M, Menchaca E, Nava JF, Nava N, Orellana JE, Ponce S. "Pulpotomía infantil utilizando ungüento oftálmico como método alternativo al uso del formocresol". Revista Latinoamericana de Ortodoncia y Odontopediatría "Ortodoncia.ws" edición electrónica mayo 2010. Obtenible en: www.ortodoncia.ws. Consultada 12 de Diciembre de 2012.

27. Flaitz CM, Barr ES, Hicks MJ. Radiographic evaluation of pulpal therapy for primary anterior teeth. J Dent Child 1989;56:182-5.

28. American Dental Association. 2009 Survey of Dental Fees. Chicago, Ill: ADA 2009;1-255.

29. Aminabadi NA, Farahani RM, Gajan EB. A clinical study of formocresol pulpotomy versus root canal therapy of vital primary incisors. J Clin Pediatr Dent 2008;32:211-4.

30. Casas MJ, Kenny DJ, Johnston DH, et al. Outcomes of vital primary incisor ferric sulfate pulpotomy and root canal therapy. J Can Dent Assoc 2004;70:34-8. 